\title{
Variability Survey of $\omega$ Centauri in the Near-IR: Period-Luminosity Relations
}

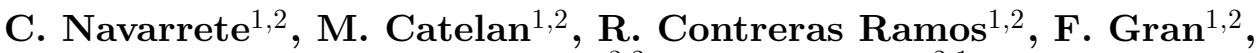 \\ J. Alonso-García ${ }^{3,2}$ and I. Dékány ${ }^{2,1}$ \\ ${ }^{1}$ Instituto de Astrofísica, Pontificia Universidad Católica de Chile, \\ Av. Vicuña Mackenna 4860, 782-0436 Macul, Santiago, Chile \\ email: [cnavarre, mcatelan] Castro.puc.cl \\ ${ }^{2}$ Millennium Institute of Astrophysics, Santiago, Chile \\ ${ }^{3}$ Unidad de Astronomía, Fac. Cs. Básicas, Universidad de Antofagasta, \\ Avda. U. de Antofagasta 02800, Antofagasta, Chile
}

\begin{abstract}
Centauri is by far the most massive globular star cluster in the Milky Way, and possibly the remnant of a dwarf galaxy. As such, it contains a large number of variable stars of different classes. Here we report on an extensive, wide-field time-series study of $\omega$ Cen in the $J$ and $K_{\mathrm{S}}$ bands, which has allowed us to study the near-IR period-luminosity relations for different variability classes, including the first such relations for the SX Phoenicis stars.
\end{abstract}

Keywords. stars: variables: RR Lyrae, stars: variables: SX Phoenicis, stars: variables: general, globular clusters: individual: $\omega$ Centauri, infrared: stars, surveys, stars: horizontal-branch

$\omega$ Centauri (NGC 5139) is by far the most massive globular star cluster in the Milky Way, and has even been suggested to be the remnant of a dwarf galaxy. As such, it contains a large number of pulsating variable stars. Among them, RR Lyrae (RRL) and Type II Cepheids (T2Cs) are well-known for following tight period-luminosity (PL) relations in the near-IR (Catelan \& Smith 2015). SX Phoenicis stars (SX Phe) are also expected to follow PL relations, but no such relations have heretofore been reported in the near-IR. $\omega$ Cen is one of the best targets for this purpose, as it has a rich variable star content, hosting at least 270 pulsating stars, including T2Cs, RRL, and SX Phe stars.

A deep, wide-field, near-IR variability survey of $\omega$ Cen was carried out by our team using ESO's 4.1m VISTA telescope. Our time-series data comprise 42 and 100 epochs in $J$ and $K_{\mathrm{S}}$, respectively. Figure 1 shows the $K_{\mathrm{S}},\left(J-K_{\mathrm{S}}\right)$ color-magnitude diagram for the cluster (based on data for the innermost 10 arcmin), with the detected variable stars overplotted (based on our entire dataset, covering $1.1 \times 1.5 \mathrm{deg}^{2}$ around the cluster center). This unique dataset has allowed us to derive complete $K_{\mathrm{S}}$-band light curves for more than 200 variable stars in the cluster's field.

Using this dataset, Navarrete et al. (2015) reported an updated census on the number, variability type and membership of all but 4 of the known RRL stars in $\omega$ Cen's field, and also discovered four new RRL. Moreover, reference Oosterhoff I and II loci in the Bailey (period-amplitude) diagram were derived for the $J$ and $K_{\mathrm{S}}$ bands, in addition to expressions relating the light-curve amplitudes in the $V, J$, and $K_{\mathrm{S}}$ bandpasses.

This unprecedented variable star catalogue also allow us to perform a detailed analysis of the near-IR PL relations for different variability classes, including T2Cs, SX Phe, and RRL stars (Navarrete et al., in prep.). Based on our intensity-averaged magnitudes, and using calibrated PL relations from the literature (Matsunaga et al. 2006 for T2Cs, Alonso-García et al. 2015 for RRLs), an updated distance modulus for the cluster was also 


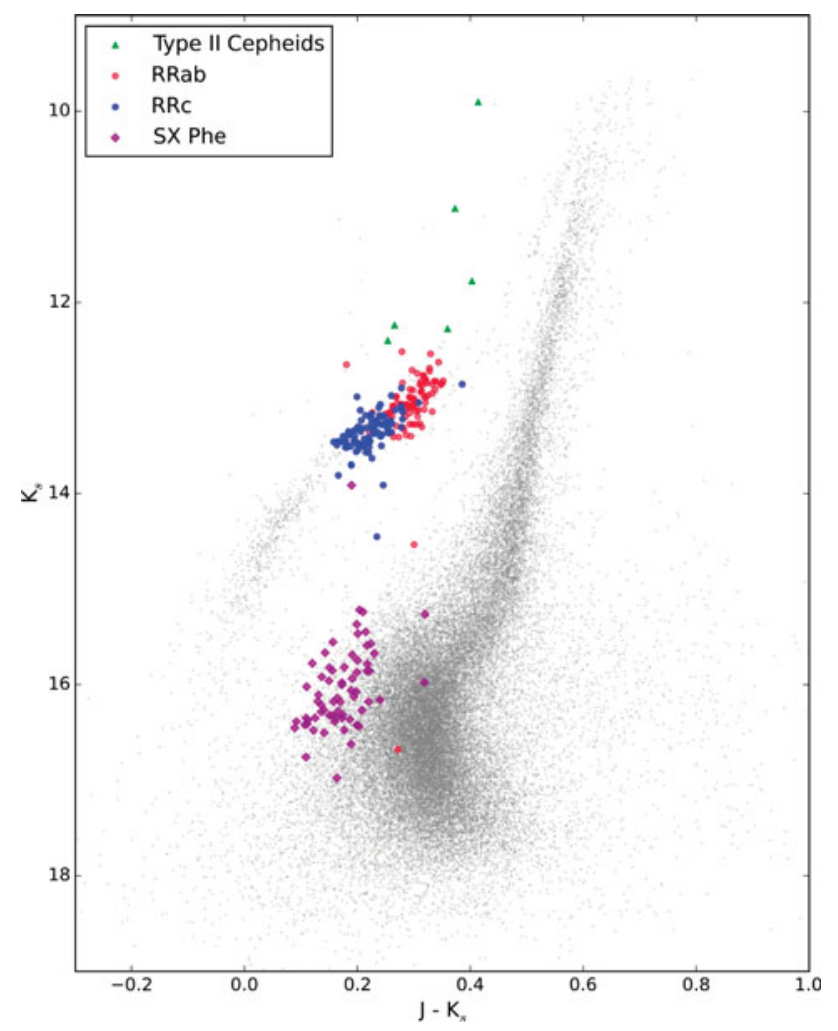

Figure 1. Near-IR CMD showing the non-variable $\omega$ Centauri stars in the cluster's innermost region $\left(11 \operatorname{arcmin}^{2}\right.$, grey dots $)$. Variable stars over the entire cluster field $\left(1.1 \times 1.5 \mathrm{deg}^{2}\right)$ are marked with green triangles (T2Cs), red and blue circles (RRab and RRc, respectively), and purple diamonds (SX Phe).

obtained. For SX Phe stars, fundamental-mode candidates (Olech et al. 2005; McNamara 2011) have been considered to derive, for the first time, calibrated near-IR PL relations.

\section{Acknowledgements}

This project is supported by FONDECYT grants \#1130373 and \#1141141, and by Basal-CONICYT grant PFB-06, awarded to the Centro de Astrofísica y Tecnologías Afines (CATA). Additional support is provided by the Ministry for the Economy, Development, and Tourism's Iniciativa Científica Milenio through grant IC 120009, awarded to the Millennium Institute of Astrophysics (MAS). C.N. acknowledges additional support from a SOCHIAS grant through Gemini/CONICYT Project \#32140015, and CONICYT-PCHA/Doctorado Nacional 2015-21151643.

\section{References}

Alonso-García, J., Dékány, I., Catelan, M. et al. 2015, AJ, 149, 99

Catelan, M. \& Smith, H. A. 2015, Pulsating Stars (Wiley-VCH)

Matsunaga, N., Fukushi, H., Nakada, Y., et al. 2006, MNRAS, 370, 1979

McNamara, D. H. 2011, AJ, 142, 110

Navarrete, C., Contreras Ramos, R., Catelan, M., et al. 2015 A\&A, 577, 99

Olech, A., Dziembowski, W. A., Pamyatnykh, A. A., et al. 2005, MNRAS, 363, 40 